\title{
Quelles sont les activités réellement réalisées par les chefs d'établissement dans le cadre d'un leadership pédagogique? Revue de la littérature
}

\author{
Sylvie Bos et Sébastien Chaliès \\ Université de Toulouse
}

Cet article rend compte de la littérature relative aux activités réalisées au quotidien par les chefs d'établissement dans l'exercice de leur leadership pédagogique. Il permet de mettre en exergue que si "l'effet chef d'établissement" sur l'amélioration de la réussite des élèves apparaît comme bien étudié, il n'en reste pas moins peu abordé sous l'angle des activités réellement menées au quotidien dans les établissements scolaires. Sur la base de la description des principales activités recensées dans la littérature du domaine, comme celles relatives à la définition d'une politique d'établissement, à la mobilisation et la formation des enseignants, à l'influence sur les pratiques pédagogiques ou encore à l'utilisation stratégique d'aspects structurels ou organisationnels propres à la vie de l'établissement, l'article ouvre de nouvelles pistes de travaux.

\section{Introduction}

Bien que le concept d'activité soit sans doute encore à considérer comme un concept aux contours flous en sciences humaines et sociales (Barbier \& Durand, 2003), tant les définitions sont multiples et diverses, dans le cadre de cet article, le choix a été fait d'assimiler l'activité des chefs d'établissement à ce qu'ils font, ou disent de ce qu'ils auraient voulu faire sans pouvoir véritablement le faire dans les établissements scolaires. En ce sens, une définition de l'activité proche de celle proposée par Clot en clinique de l'activité (Clot, 2008) est adoptée. L'activité des chefs d'établissement sera donc considérée comme, pour partie, "réalisée», et à ce titre, observable, mais aussi comme comprenant ce qui a été envisagé, mais n’a pas pu être réalisé.

L'activité des chefs d'établissement est soumise à un ensemble de prescriptions et de contraintes qui varient fortement d'un système éducatif à l'autre (Pont, Nusche, \& Moorman, 2008). Toutefois, quel que soit le système éducatif, une part significative de cette activité est typiquement consacrée à chercher à améliorer la réussite des élèves (Bossert, Dwyer, Rowan, \& Lee, 1982; Hallinger, 2011a; 
Leithwood, Harris, \& Hopkins, 2008; Neumerski, 2013). En effet, outre le fait de diriger administrativement et humainement l'établissement, les chefs d'établissement réalisent des activités contributives d'un «leadership pédagogique» (Hallinger, 2011b) dont la finalité première est l'amélioration de la réussite des élèves. Largement sous-tendu par de multiples prescriptions institutionnelles (Cour des comptes, 2010; Inspection générale de l'administration de l'éducation nationale et de la recherche [IGAENR], 2010; Organisation de coopération et de développement économiques [OCDE], 2014), le déploiement d'un tel leadership n'en reste pas moins souhaité et valorisé par les chefs d'établissement eux-mêmes (Progin \& Gather Thurler, 2017). On comprend dès lors l'intérêt de se questionner sur les activités qu'ils et elles engagent pour déployer un tel leadership, et par-là même, œuvrer à l'amélioration de la réussite des élèves, dans un rôle qui leur est propre: celui de prescrire et de structurer en partie le travail des autres, notamment celui des enseignants (Gather Thurler \& Maulini, 2007). C'est donc à ce niveau que se situe l'objet premier de cette revue de littérature. À partir du recueil et de l'étude des travaux scientifiques récents, cet article s'efforce de recenser, d'analyser et mettre en exergue les principales activités réalisées par les chefs d'établissement dans le cadre d'un leadership pédagogique.

Avant d'entrer dans l'explicitation de la méthodologie adoptée pour mener à bien cette revue de littérature et dans la description des principaux résultats obtenus, il est à noter que parmi les résultats qui font consensus dans la communauté scientifique, celui selon lequel il existe un «effet chef d'établissement» sur la réussite des élèves, autrement dit un effet de l'activité des chefs d'établissement sur cette réussite, est tout à fait significatif. Pour bon nombre d'auteurs (par exemple: Day et al., 2010; Jacobson, 2011; Muijs, 2011; Supovitz, Sirinides, \& May, 2010) la réussite des élèves est en effet directement influencée par les activités réalisées par les chefs d'établissement. Menée à partir des travaux publiés entre 1980 et 2010, la revue de littérature réalisée par Hallinger (2011b) est à ce titre sans équivoque. Plus en détails, certains auteurs soulignent que cet effet varie selon la nature du leadership mis en ouvre par les chefs d'établissement au quotidien dans les établissements scolaires. Ainsi, Murphy, Vriesenga et Storey (2007) précisent que plus le leadership est de nature "pédagogique», plus il influe sur les résultats des élèves. Pour ces auteurs, cet effet est lié au fait que, dans ce type de leadership, les chefs d'établissement sont principalement préoccupés par le "cœur de leur mission", à savoir les apprentissages des élèves, et qu'ils et elles centrent leurs activités sur l'optimisation des modalités d'enseignement des enseignants, le respect des programmes, ou encore les modalités et la nature des évaluations proposées aux élèves. Dans ce type de leadership, les autres dimensions, relatives à la gestion administrative et financière de l'établissement scolaire, sont, dans le même temps, directement placées au service de la pédagogie. Dans le même ordre d'idées, Robinson, Lloyd et Rowe (2008) montrent, en s'appuyant sur les travaux réalisés dans le champ, que la réussite des élèves est d'autant plus élevée que les chefs d'établissement s'engagent dans des activités visant l'amélioration des pratiques pédagogiques des enseignants. 


\section{Méthode adoptée pour réaliser la revue de littérature}

La méthode adoptée se compose de trois étapes successives: (i) l'élaboration d'une stratégie de recueil des publications scientifiques à partir de moteurs de recherche électroniques, (ii) la définition de critères d'inclusion/exclusion pour sélectionner les publications scientifiques à retenir et, enfin, (iii) l'analyse de ces publications. Ces trois étapes sont détaillées ci-après.

\section{Élaboration d'une stratégie de recueil des \\ publications scientifiques}

Le recueil des publications scientifiques exploitées pour construire la revue de littérature a été effectué à l'aide des principaux moteurs de recherche nationaux et internationaux en sciences de l'éducation (ERIC, Science Direct, Academic Search Premier et Cairn info). Ce recueil a été effectué sur la période 2007-2017.

Cette première étape a été initiée par un travail de clarification des mots clés et des terminologies les plus utilisées dans le champ. Au sein des travaux anglophones, l'activité de direction menée par les chefs d'établissement est ainsi usuellement assimilée au «leadership», ou au "management» (Hallinger, 2011b). Au sein des travaux francophones, le concept de leadership est aussi largement utilisé même si, parfois, le concept de "pilotage» des établissements scolaires lui est substitué (voir pour plus de détails: Endrizzi \& Thibert, 2012; Gather Thurler, Pelletier, \& Dutercq, 2015). Un recensement des travaux menés dans le champ a permis de constater que la préoccupation d'étudier les activités réalisées par les chefs d'établissement pour tenter d'améliorer la réussite des élèves apparaissait dans les années 1980 sous la terminologie d' «instructional management» (Bossert et al., 1982). Cette terminologie a été progressivement remplacée par celle d' "instructional leadership" et d'ueducational leadership» (Hallinger, 2011b), puis notamment en Europe, par celle de «leadership for learning» (MacBeath \& Cheng, 2008). Dans la plupart des travaux, le «leadership pédagogique» («educational leadership») est défini comme un ensemble comprenant «le rôle, le comportement et/ou l'impact des chefs d'établissement sur les apprentissages des élèves» (Hallinger, 2013, p. 550). À ce niveau, il est à noter qu'un certain nombre de contraintes ralentissent, pour ainsi dire, le développement de ce champ de recherche. Dans plusieurs pays européens, l'étude des directions des établissements scolaires ne relève pas en effet d'un champ de recherche spécifiquement identifié. Les «administrative sciences» développées au sein des pays anglophones n'ont pas, par exemple, d'équivalent dans les classifications scientifiques francophones comme celle du Conseil National des Universités en France. Ce constat permet sans doute de comprendre pourquoi, bien que la situation évolue sous l'influence des apports d'autres pays de la francophonie (Ducros \& Amigues, 2014; Letor \& Garant, 2014; Progin \& Gather Thurler, 2012), les travaux menés sur le leadership des chefs d'établissement se diffusent mais restent peu 
utilisés en France (Derouet $\&$ Normand, 2014). Une autre contrainte naît aussi de la multiplicité des définitions associées au concept de "leadership» dans la littérature. Cette multiplicité rend en effet difficile de mener à bien une revue de la littérature à partir de ce seul concept. De la même manière, alors que certains travaux associent le leadership aux seuls chefs d'établissement, d'autres considèrent que tout personnel de l'établissement scolaire en charge d'une responsabilité est engagé dans une activité de leadership (Harris \& Spillane, 2008; Progin \& Gather Thurler, 2010; Leithwood, Mascall, Strauss, Sacks, Memon, \& Yashkina, 2007). La littérature distingue ainsi, dans l'étude du leadership, les «leaders institutionnels» (chefs d'établissement, inspecteurs, doyens, coordonnateurs de discipline), également désignés sous le terme de «leaders formels», et les «leaders informels» (tels que les enseignants, les porteurs de projets).

Finalement, compte tenu de l'objet d'étude adopté et des précisions conceptuelles apportées en amont, plusieurs mots clés ont été retenus et exploités au sein des moteurs de recherche pour effectuer le recueil des publications scientifiques. Dans la littérature anglophone les principaux mots clés retenus ont été: "educational leadership», "instructional leadership», "leadership for learning» et «distributed leadership». Dans la littérature francophone les mots clés retenus ont été: "chef d'établissement», «"pilotage», «leadership pédagogique» et «leadership éducatif». L'utilisation de ces mots clés a permis de recueillir un total de 2656 publications scientifiques (Tableau 1).

\section{Tableau 1: Répartition du nombre de publications scientifiques recueillies en} fonction des moteurs de recherche exploités

\begin{tabular}{|c|c|c|}
\hline $\begin{array}{c}\text { Moteurs de recherches } \\
\text { exploités }\end{array}$ & $\begin{array}{c}\text { Keys words } \\
\text { «educational leadership» or/and } \\
\text { «instructional leadership» or/ } \\
\text { and «leadership for learning» or/ } \\
\text { and «distributed leadership» and } \\
\text { "principal» }\end{array}$ & $\begin{array}{c}\text { Mots clés } \\
\text { "chef d'établissement» } \\
\text { et/ou «pilotage» } \\
\text { et/ou «leadership pédagogique» } \\
\text { et/ou «leadership éducatif» }\end{array}$ \\
\hline ERIC & 1112 & 3 \\
\hline Academic Search Premier & 888 & 0 \\
\hline Sciences Direct & 596 & 5 \\
\hline Cairn Info & 15 & 45 \\
\hline Total & 2611 & 37 \\
\hline
\end{tabular}

Définition des critères d'inclusion/exclusion pour sélectionner les publications scientifiques significatives

Parmi l'ensemble des publications initialement recensées, le choix a tout d'abord été fait de sélectionner celles assimilables à des «revues de littérature» ou comportant une mise en perspective historique des travaux réalisés dans le domaine (12 au total). Pour les autres publications, une sélection a été réalisée, 
à partir de l'exploitation de critères d'inclusion et d'exclusion établis en fonction de l'objet de la revue de littérature (Tableau 2).

\section{Tableau 2: Critères d'inclusion et d'exclusion des publications scientifiques initia- lement recueillies}

\begin{tabular}{|c|c|}
\hline Critères d'inclusion & Critères d'exclusion \\
\hline $\begin{array}{l}\text { Publications ayant comme objet principal le } \\
\text { rôle pédagogique des chefs d'établissement } \\
\text { et/ou } \\
\text { Publications citant des activités constitutives } \\
\text { du rôle pédagogique des chefs d'établissement } \\
\text { et } \\
\text { Publications réalisées entre } 2007 \text { et } 2017\end{array}$ & $\begin{array}{l}\text { Publications théoriques sur les modélisations du } \\
\text { leadership } \\
\text { Publications centrées sur les caractéristiques person- } \\
\text { nelles du chef d'établissement ou sur les valeurs qu'il } \\
\text { ou elle véhicule } \\
\text { Publications relatives aux politiques publiques, aux } \\
\text { politiques éducatives } \\
\text { Publications relatives aux liens avec les partenaires } \\
\text { extérieures et les parents d'élèves }\end{array}$ \\
\hline
\end{tabular}

Pour sélectionner les publications à partir de ces critères d'inclusion et d'exclusion, une lecture de leur résumé (ou abstract) a été réalisée. Sur l'ensemble des publications initialement recueillies seules 45 ont été retenues car, en leur sein, l'activité des chefs d'établissement était décrite et/ou analysée par les chercheurs et/ou les chefs d'établissement eux-mêmes. Seules ces publications ont été étudiées exhaustivement. Leur bibliographie a été croisée avec les publications recueillies de façon à compléter le recueil des publications scientifiques significatives dans le domaine. La plupart des 77 publications finalement retenues sont des articles de revues scientifiques appartenant au champ des sciences de l'éducation, des sciences de gestion, de la sociologie ou des administrative sciences. Dans une moindre mesure, des ouvrages ou chapitres d'ouvrages ainsi que des publications institutionnelles ont été sélectionnés pour être analysés.

\section{Analyse des publications sélectionnées}

Les publications sélectionnées ont enfin été analysées afin de parvenir à construire les résultats structurant la revue de littérature. Pour ce faire, une analyse qualitative descriptive, intentionnellement non interprétative (Strauss \& Corbin, 1990), a été réalisée. Chaque résultat, ou partie de résultat, est à ce titre le fruit d'une catégorisation, ou d'une sous-catégorisation, suite à l'analyse de chacune des publications sélectionnées. Par principe, une nouvelle catégorie, ou souscatégorie, a été créée quand les éléments proposés par la publication analysée ne pouvaient être classés au sein d'une catégorie, ou d'une sous-catégorie, déjà existante. 


\section{Résultats}

Nos résultats montrent, en premier lieu, que peu de travaux ont réellement pour objet premier d'étude l'activité réalisée par les chefs d'établissement dans les établissements scolaires (Matringe, 2012; Raelin, 2017). Ce constat fait d'ailleurs dire à certains auteurs qu'il existe de ce point de vue une "black box» (Hallinger, 2011b). De nos jours, peu de travaux décrivent et analysent effectivement la ou les activités réellement menées par les chefs d'établissement pour exercer leur leadership pédagogique (Neumerski, 2013). De la même manière, même si «ce qui doit se faire» et "pourquoi il est nécessaire de le faire» semble assez travaillé, la manière de procéder paraît bien plus floue, sans doute parce qu'assujettie au contexte, à la personnalité du chef d'établissement et à sa conception éducative (Mauny, 2010). Paradoxalement, nos résultats montrent qu'il existe toutefois un intérêt scientifique à établir les liens de causalité entre la nature des activités des chefs d'établissement et l'amélioration ou non de la réussite des élèves. En suivant, ce sont ces principales activités identifiées dans la littérature comme participant d'un leadership pédagogique, c'est-à-dire participant de la recherche d'amélioration de la réussite des élèves, qui sont détaillées selon trois principales catégories: les activités de définition et mise en œuvre d'une politique éducative au sein de l'établissement scolaire, les activités de gestion des ressources humaines et enfin, les activités relatives aux aspects matériels et structurels de l'établissement.

Avant d'entrer dans les résultats obtenus, notons que, dans une large majorité des travaux recensés et étudiés, les effets des activités menées par les chefs d'établissement sur l'amélioration de la réussite des élèves sont qualifiés d' «indirects». Il est rare, en effet, que les chefs d'établissement exercent leurs activités directement auprès des élèves. Ils et elles impactent donc la réussite de ces derniers en influençant plutôt divers aspects du fonctionnement de l'établissement, tels que les pratiques pédagogiques des enseignants en classe (Hallinger, 2011b; Supovitz et al., 2010), les dynamiques collectives des équipes enseignantes (Pelletier, Colerette, \& Turcotte, 2015), ou encore les conditions de travail au sein de l'établissement scolaire (Leithwood et al., 2008). Les travaux scientifiques qui étudient les activités engagées par les chefs d'établissement dans le cadre d'un leadership pédagogique sont ici regroupés.

\section{Activités réalisées pour définir et mettre en œuvre la politique éducative de l'établissement scolaire}

Les activités de définition et de mise en œuvre de la politique éducative de l'établissement scolaire sont pointées comme le premier levier permettant aux chefs d'établissement d'améliorer la réussite des élèves (Hallinger, 2011a; Robinson et al., 2008). À partir d'un travail visant à dégager les dimensions génériques des métiers de direction d'établissement public, Barbier (2011) montre que, parmi ces activités celle consistant à communiquer oralement avec les autres personnels 
de l'établissement est tout particulièrement significative. De la même manière, Tourmen, Mayen et Samrany (2011) soulignent que les activités engagées pour définir et de mettre en œuvre une politique éducative sont «essentiellement langagières». Ces auteurs précisent ainsi que les chefs d'établissement s'engagent régulièrement dans une communication spécifique avec les autres personnels, afin de "prendre des informations» sur l'environnement ou le fonctionnement de l'établissement d'une part, et de "qualifier» d'autre part, c'est-à-dire valider une prise de décision, ou porter un jugement de valeur sur une situation ou un événement. Dans le même ordre d'idées, Hallinger (2011a) montre que les chefs d'établissement sont amenés à prendre des décisions quotidiennes, comme, par exemple, engager ou non des projets pédagogiques, décider de l'allocation des ressources, ou encore définir ce qui est acceptable en termes de comportement pour un enseignant ou un élève. Ces activités, largement décisionnelles, conduisent les chefs d'établissement à définir ce qui est important au sein de la culture de l'établissement et constituent ainsi des activités d'orientation et de mise en œuvre de la politique éducative à destination des autres personnels.

$\mathrm{Au}$ sein des travaux menés dans certains pays anglo-saxons, les activités engagées par les chefs d'établissement autour de la politique éducative de leur établissement sont souvent pensées à partir des concepts de "vision et buts» («vision and goals») (voir par exemple la méta analyse réalisée par Robinson et al., 2008). Le concept de «vision» est exploité pour rendre compte des activités menées par les chefs d'établissement lorsqu'ils anticipent la direction à donner à la politique éducative de l'établissement. Le concept de «buts» renvoie, quant à lui, aux activités menées pour définir les objectifs et les actions qui vont permettre de réaliser cette vision (Hallinger, 2011a). La notion de "valeurs» est également très présente dans les études liant les activités des chefs d'établissement et l'amélioration de la réussite des élèves (Hallinger, 2011a; Robinson et al., 2008; Roegman, Hatch, \& Riehl, 2012). À partir de ces concepts, dans un registre a priori idéel, se dégagent des activités menées par les chefs d'établissement afin de définir et mettre en ouvre une politique éducative. Hallinger souligne à ce titre que les valeurs sont d'autant plus présentes dans l'orientation des activités des chefs d'établissement que ceux-ci, face à une situation complexe, ne disposent pas d'éléments d'informations objectifs suffisants. Robinson (2010) note par ailleurs que les chefs d'établissement expérimentés mettent en avant un certain nombre de valeurs lorsqu'ils et elles cherchent à maintenir les relations entre les enseignants, les parents et les élèves. Dans le prolongement, Roegman et al. (2012) notent que les choix singuliers en matière de politique éducative portés par les valeurs des chefs d'établissement se retrouvent potentiellement dans des activités telles que, par exemple, la valorisation de certaines actions lors de cérémonies officielles, la prise de position pour l'inclusion d'élèves en situation de handicap, ou encore, lors de gestion de conflits.

Finalement, nos résultats montrent que les chefs d'établissement cherchent à définir et mettre en œuvre une politique éducative au sein de leur établissement 
par l'engagement d'activités pour la plupart langagières. Ces activités consistent généralement à échanger des informations, à émettre des jugements, à définir des objectifs, à prendre position, ou encore à favoriser la réalisation d'actions éducatives en conformité avec leurs valeurs.

Activités réalisées pour gérer les ressources humaines au sein de l'établissement scolaire

Parmi toutes les activités engagées par les chefs d'établissement pour améliorer la réussite des élèves, celle consistant à gérer les ressources humaines est tout particulièrement significative. Dans une très large majorité, les chefs d'établissement considèrent en effet que l'amélioration de la réussite des élèves dépend essentiellement de la qualité du travail pédagogique engagé par chaque enseignant au sein de sa classe (Maulini \& Progin, 2014). C'est là une représentation en adéquation avec les conclusions avancées par les travaux menés sur «l'effet chef d'établissement" qui mettent en avant que, parmi tous les éléments influençant la réussite des élèves, la nature des pratiques enseignantes occupe une place centrale (Hallinger, 2011b; Pelletier et al., 2015; Supovitz et al., 2010). Sur la base de ce constat, certains travaux considèrent donc que les activités de "gestion des ressources humaines», c'est-à-dire ayant comme préoccupation première de mobiliser les enseignants et de les accompagner dans leur développement professionnel, sont essentielles dans le cadre d'un leadership pédagogique (Bravo, 2008; Gather Thurler et al., 2011; Printy, Marks, \& Bowers, 2009). Ci-après un effort de recensement et d'explicitation de ces activités est réalisé.

\section{Mobiliser les enseignants pour améliorer la réussite des élèves}

Selon les systèmes éducatifs, les prérogatives des chefs d'établissement en termes de gestion des ressources humaines varient fortement, y compris au sein de l'Union Européenne (Salamé Sala \& Dutercq, 2015). Dans certains pays, comme par exemple la France, les chefs d'établissement n'ont pas la possibilité de participer au recrutement des enseignants affectés dans leur établissement et influent ensuite assez peu sur leur évolution de carrière et leur rémunération (Barrère, 2013; Brest 2011, Obin, 2012). Dans la plupart des cas, et plus particulièrement dans le second degré, les chefs d'établissement sont placés institutionnellement dans une posture d'autorité vis-à-vis des enseignants. Ils en sont les supérieurs hiérarchiques (Pont et al., 2008). Cependant, les interactions entre les chefs d'établissement et les enseignants reflètent les contradictions de systèmes éducatifs au cour desquels des «tensions» existent entre l'autonomie volontairement donnée aux établissements scolaires et l'effort de centralisation des prises de décisions (Dutercq, 2015). La plupart des travaux qui s'intéressent à ces interactions mettent en avant que les activités réalisées par les chefs d'établissement sont en réalité assez peu marquées par des prises de position hiérarchique ou de contrôle. Elles sont généralement empreintes d'une vision hiérarchique relativement démocratique, au sein de laquelle une réelle coopération avec les enseignants est souhaitée (Maulini \& Progin 2013). 
Certains auteurs soulignent que, même si l'application des politiques éducatives fait partie de l'identité professionnelle des chefs d'établissement (Lessard, Kamanzi, \& Larochelle, 2008; Normand, 2015), ces derniers étant souvent eux-mêmes issus du corps enseignant, ils s'en écartent parfois pour "protéger» les enseignants, ou éviter d'entrer en conflit avec eux (Progin \& GatherThurler, 2012). Ce type de positionnement tient aussi au fait qu'ils préferent être prudents dans l'exercice de leur fonction hiérarchique. Un positionnement inadapté pourrait leur faire courir le risque d'une organisation solidaire des enseignants en contre-pouvoir, organisation qui, in fine, pourrait empêcher tout changement au sein de l'établissement scolaire et dégrader le climat de travail (Gather Thurler, Denecker, \& Meyer, 2014). Face à ce risque, certains travaux mettent en évidence que les chefs d'établissement déploient parfois des stratégies de détour avec les enseignants pour parvenir à leurs fins (Progin \& GatherThurler, 2012). Poirel et Yvon (2012) décrivent, par exemple, une situation où un enseignant ne parvient pas à endiguer des manifestations de violence qui ont lieu spécifiquement dans sa classe. Ils montrent que le chef d'établissement, dans ce cas, s'engage avec prudence dans des activités plus ou moins simultanées d'affirmation d'une autorité vis-à-vis des élèves et de régulation des pratiques de l'enseignant.

Il est à noter que peu nombreux sont encore les travaux qui étudient réellement les activités d'interaction des chefs d'établissement avec les enseignants. Les travaux se centrent le plus souvent sur la nature des interactions, qui sont alors présentées comme pouvant être empreintes de confiance ("relational trust»), de respect et d'enthousiasme, grâce notamment à une volonté de cohésion et d'ouverture d'esprit de la part des chefs d'établissement (Leithwood et al., 2008; Robinson, 2010). Dans certaines circonstances, ces interactions apparaissent comme chargées de convivialité, de complicité ou d'amitié (Letor, 2010). Enfin, certains travaux mettent en avant que la nature de ces interactions est le plus souvent marquée par une vision éthique des chefs d'établissement vis-à-vis des enseignants (Brest, 2011). Certains auteurs soulignent que les interactions entre les chefs d'établissement et les enseignants ne peuvent toutefois s'inscrire continument dans un mode de fonctionnement "participatif» (Baluteau, 2009a). Ce mode fluctue en effet fortement en termes de directivité (Brest, 2011; Hassani \& Meuret, 2010), et ce notamment, en fonction de l'avancée dans la carrière des chefs d'établissement (Progin, 2015).

Dans le même temps, si les chefs d'établissement sont animés d'une volonté d'adhésion des enseignants au fonctionnement démocratique qu'ils cherchent à instaurer, ils et elles n'en sont pas moins responsables des résultats de leur établissement. Ces résultats étant eux-mêmes directement dépendants des résultats quantifiables des élèves (résultats aux examens, évaluations standardisées, moyennes trimestrielles, taux et nature des orientations des élèves, etc.), les chefs d'établissement sont donc naturellement amenés à investir le champ des pratiques d'évaluation, pour parvenir à influer, à plus ou moins court terme, 
sur les indicateurs chiffrés de réussite de leur établissement (Barrère, 2013). Ils et elles peuvent donc être amenés, dans une logique de pilotage par les résultats, à faire des choix auxquels n'adhèrent pas les enseignants, comme, par exemple, s'opposer à des projets qu'ils jugent sans impact sur la réussite aux examens (Barrère, 2009).

Lorsque leur statut rend possible la visite des enseignants dans les classes, comme par exemple en Suisse, ou encore l'élaboration avec les enseignants de programmes d'enseignement spécifiques, comme par exemple aux États-Unis d'Amérique, les chefs d'établissement s'engagent dans ces activités qui sont considérées comme contributives de l'amélioration de la réussite des élèves (Robinson et al., 2008). Les chefs d'établissement peuvent en effet, dans ce type de contexte, exercer une influence directe sur les pratiques pédagogiques, même s'ils préferent souvent rester à «l'extérieur» de la classe, et déléguer l'appréciation des activités d'enseignement aux acteurs du système éducatif représentant des disciplines (inspecteurs, doyens, etc.), qu'ils jugent plus compétents (Progin $\&$ Gather-Thurler, 2012). Il est à noter que c'est lorsque les chefs d'établissement considèrent qu'aucune coopération n'apparait entre enseignants, qu'ils s'engagent dans des activités relatives aux pratiques pédagogiques dans la classe (Perrenoud, 2015). Ne pouvant ou ne souhaitant pas pénétrer dans les classes, ils cherchent alors à mobiliser les enseignants lors de réunions collectives, au cours desquelles leurs interventions au sujet des pratiques pédagogiques semblent plus facilement audibles (Progin \& Gather Thurler, 2012).

Outre les échanges au sujet des pratiques pédagogiques, la mobilisation des enseignants peut se réaliser par l'engagement des chefs d'établissement dans la coordination et l'harmonisation des évaluations et des pratiques pédagogiques. Ils peuvent ainsi décider du rythme et du volume des évaluations, de l'étendue et la sévérité des règles et des sanctions au sein de l'établissement, ou encore, des lignes directrices du projet d'établissement et des projets disciplinaires qui en découlent (Barrère, 2009; Maulini \& Progin 2014). Les chefs d'établissement se saisissent des pratiques d'évaluation comme objet de discussion afin que, par voie de retour, les pratiques pédagogiques soient interrogées. Certains auteurs (par exemple: Normand \& Derouet, 2011; Perrenoud, 2015) considèrent en effet que la réflexion sur les pratiques évaluatives constitue un levier pour les chefs d'établissement qui, en cherchant avec les enseignants à les harmoniser, visent en réalité une optimisation des pratiques pédagogiques et, par-là même, une amélioration de la réussite des élèves.

Certains auteurs considèrent par ailleurs que les difficultés scolaires rencontrées par les élèves peuvent être exploitées par les chefs d'établissement pour mobiliser les enseignants et les inviter à interroger leurs pratiques pédagogiques. Progin et Gather Thurler (2012) montrent que, dans ce cas, les chefs d'établissement adoptent des stratégies différentes, soit en s'engageant dans une logique d'externalisation de la remédiation (par la mise en place, par exemple, de dispositifs de soutien ou d'appui), soit en cherchant plutôt à améliorer l'organisation 
interne, par une meilleure coordination du travail au sein du collectif enseignant. Enfin, Leitwhood et al. (2007), soulignent que les chefs d'établissement identifient, en fonction des compétences de chacun, les enseignants sur lesquels ils pourraient s'appuyer, et les encouragent à prendre davantage de responsabilités au sein de l'établissement, afin de les mobiliser.

Une large majorité des travaux du domaine s'accordent finalement pour déclarer que les chefs d'établissement engagent des activités visant à mobiliser les enseignants. Cette mobilisation s'effectue le plus souvent par un travail plus ou moins direct sur les pratiques pédagogiques engagées et leurs améliorations possibles. Ces activités, très dépendantes du contour des missions définies au sein de chaque système éducatif, sont toujours guidées par un souci d'adhésion des enseignants. Elles sont le plus souvent réalisées au détour d'autres activités, via des objets intermédiaires, tels que les évaluations ou les difficultés scolaires des élèves.

\section{Contribuer au développement professionnel des enseignants}

Parmi les activités engagées par les chefs d'établissement en termes de gestion des ressources humaines pour accroître la réussite des élèves, celles nourries par l'intention de contribuer au développement professionnel des enseignants sont aussi mises en avant par certains travaux du domaine (voir par exemple: Hallinger, 2011a; Hassani \& Meuret, 2010; Perrenoud, 2015) et en particulier par ceux étudiant les conditions du développement du travail en équipe enseignante (Baluteau, 2009b; Barrère, 2009; Ducros \& Amigues 2014; Kools \& Stoll 2016). Ces travaux s'accordent sur le fait qu'en favorisant les conditions du développement professionnel, en sélectionnant les modalités de formation et en créant une culture de l'apprentissage au sein de l'établissement, les chefs d'établissement contribuent à optimiser les pratiques pédagogiques des enseignants et par là-même participent à l'amélioration de la réussite des élèves (Hallinger, 2011a; Leithwood et al., 2007; Normand \& Derouet, 2011; Progin \& Gather Thurler, 2010). Ces travaux décrivent des activités de mises en place de temps de réflexion, voire de formation, pour tenter de répondre à des problématiques locales (en matière de difficultés d'élèves par exemple) ou de s'adapter à de nouvelles prescriptions institutionnelles (en matière d'usage du numérique par exemple) (Maulini \& Progin, 2014; Perrenoud, 2015).

Il est toutefois à noter que les activités engagées par les chefs d'établissement pour chercher à transformer les pratiques enseignantes ne sont pas sans poser de difficultés. Ces activités sont en effet le plus souvent adressées à un collectif d'enseignants au sein duquel les enjeux de pouvoir (Dumay, 2009), les affinités ou encore la nécessaire singularité des difficultés rencontrées au quotidien se révèlent comme autant de freins (Barrère, 2010). Si la plupart des travaux valorisent une approche du développement professionnel par le travail collectif, des études soulignent ainsi que l'activité des chefs d'établissement est plus efficace si elle est centrée sur l'individualisation des choix de formation des enseignants 
et qu'elle s'appuie sur une connaissance fine du parcours et des ressources de chacun d'entre eux (Brezicha, Bergmark, \& Mitra, 2015).

Les chefs d'établissement engagent donc des activités qui visent le développement professionnel des enseignants par la mise en place de formations individuelles ou collectives et la création de situations de travail en collectif. De façon plus structurelle, Muller et Normand (2013) soulignent que l'élément le plus déterminant pour le développement professionnel des enseignants est sans doute le fait que, au quotidien, les chefs d'établissement, par leur propre activité, autorisent la prise de risque et le questionnement chez les enseignants, ou, au contraire, cantonnent ces derniers dans des habitudes traditionnelles.

\section{Activités réalisées pour gérer les dimensions matérielles et organisationnelles de l'établissement scolaire}

Parmi les activités engagées par les chefs d'établissement pour chercher à améliorer la réussite des élèves, celles associées à l'intention d'optimiser les aspects organisationnels et matériels au sein de l'établissement scolaire sont aussi mises en avant dans la littérature scientifique. En procédant ainsi, les chefs d'établissement cherchent à améliorer les conditions de travail (Leithwood et al., 2008) et le «climat scolaire» au sein l'établissement (Baudrit \& Rich, 2013), ainsi qu'à formaliser, diriger et ordonner les activités des enseignants (Hallinger, 2011a; Sergi, 2016). Réalisées autour des dimensions matérielles et organisationnelles de l'établissement scolaire, ces activités sont engagées pour répondre à ce que Dupriez (2010) nomme des «tâches d'administration ou d'organisation pédagogique», dissociées volontairement des "tâches d'animation pédagogique». Selon cet auteur, des activités «d'administration ou d'organisation pédagogique» sont réalisées, par exemple, lors de la construction du projet d'établissement, de l'attribution des classes aux professeurs et professeurs principaux, des négociations relatives à l'ouverture d'enseignements dits optionnels, ou encore de l'administration de projets pédagogiques. Multiples, ces activités d'organisation du travail scolaire et de gestion des moyens participent potentiellement à l'amélioration de la réussite des élèves (Neumerski, 2013; Perrenoud, 2015).

Parmi les activités organisationnelles menées par les chefs d'établissement, celles relatives à la mise en œuvre de projets pédagogiques locaux (sorties scolaires, partenariats, classes à projet, etc.), peuvent être mobilisées dans le cadre d'un leadership pédagogique (Perrenoud, 2015). En organisant ce type de projets au sein de l'établissement, les chefs d'établissement se créent en effet un espace au sein duquel ils deviennent légitimes pour intervenir auprès des enseignants sur la dimension pédagogique (Barrère, 2013). Dans le même ordre d'idées, la participation au choix des manuels scolaires, pour valider la conformité aux prescriptions institutionnelles par exemple, est également associée à une activité pouvant permettre, aux chefs d'établissement, à partir d'une question matérielle, d'influer sur l'apprentissage des élèves (Poirel \& Yvon, 2012). Les travaux menés 
par Barrère $(2009$; 2013) et Baluteau (2009b) sont dans ce domaine aussi significatifs. Ces auteurs montrent que les chefs d'établissement, lorsqu'ils mènent des activités d'attribution de classes, ou de groupes, aux enseignants, ont un objectif de portée pédagogique sur le travail des élèves. Ils pointent ainsi que les chefs d'établissement sélectionnent les enseignants en fonction des caractéristiques professionnelles perçues, ou encore, en fonction des dispositifs mis en œuvre au sein de l'établissement (classes dites à examen, classes complexes à gérer, groupes de soutien, équipes harmonisées, etc.). De la même manière, ces auteurs montrent qu'une attention particulière est portée par les chefs d'établissement à la sélection des professeurs principaux et des coordonnateurs, auxquels ils attribuent des responsabilités d'encadrement intermédiaire. Baluteau (2009b) identifie également l'activité de constitution des classes et de groupes d'élèves (groupes de niveaux, hétérogènes ou équilibrés, élèves à séparer, etc.) comme particulièrement significative. Dans le même ordre d'idées, cet auteur souligne l'importance de l'activité relative à la construction du temps scolaire des élèves. En travaillant la constitution des emplois du temps hebdomadaires, les chefs d'établissement cherchent à faire évoluer l'activité pédagogique des enseignants, et à terme, à améliorer la réussite des élèves. Il montre, plus précisément, que la construction du temps scolaire est tenue, tout à la fois, par des objectifs d'optimisation des rythmes d'apprentissages des élèves et par des enjeux, parfois contradictoires, de maintien d'un climat relationnel serein avec les enseignants.

L'analyse des travaux recensés permet donc de montrer que les activités influant sur la structure et l'organisation de l'établissement scolaire sont réalisées par les chefs d'établissement afin de chercher à améliorer la réussite des élèves. Ces activités restent malgré tout encore assez peu étudiées alors même qu'elles représentent une part importante du travail quotidien des chefs d'établissement (Dupriez, 2010).

\section{Discussion: synthèse, difficultés et perspectives}

\section{Synthèse des résultats}

De façon macroscopique, cette revue de la littérature permet de mettre en exergue que de nombreuses études ont pour objet le leadership pédagogique des chefs d'établissement. Le constat peut néanmoins être dressé que, parmi ces études, seules certaines centrent leur analyse sur les activités réellement réalisées par les chefs d'établissement dans les établissements scolaires. En d'autres termes, si «l'effet chef d'établissement» sur l'amélioration de la réussite des élèves apparaît comme bien étudié, il n'en reste pas moins peu abordé sous l'angle des activités réalisées au quotidien dans les établissements scolaires.

Plus en détails, cette revue de la littérature permet de situer et détailler les principales activités réalisées par les chefs d'établissement pour chercher à 
améliorer la réussite des élèves. Parmi ces activités, certaines se révèlent comme tout particulièrement significatives: définir une politique d'établissement, influencer les pratiques pédagogiques ou encore utiliser stratégiquement des aspects structurels ou organisationnels propres à la vie de l'établissement scolaire pour mobiliser et former les enseignants. Cette revue de la littérature permet, par ailleurs, de mettre en exergue que les travaux s'accordent sur le fait que l'«effet chef d'établissement» est d'autant plus marqué que la préoccupation première de ceux-ci est celle de l'apprentissage des élèves. C'est sans doute là un résultat significatif qui permet de relativiser la vision selon laquelle un ou une chef d'établissement pourrait ne faire qu'administrer son établissement et rester à l'écart des problématiques pédagogiques. De manière complémentaire, l'analyse des travaux permet de mettre en évidence un consensus sur le caractère «indirect» de «l'effet chef d'établissement» sur l'amélioration de la réussite des élèves. Comme explicité en amont, les chefs d'établissement s'engagent en effet régulièrement dans des stratégies de détour, elles-mêmes déployées ensuite au quotidien sous la forme d'activités singulières, pour parvenir à leurs fins.

À ce niveau, il est sans doute nécessaire de souligner que, si des travaux s'accordent sur les retombées des activités des chefs d'établissement sur l'amélioration de la réussite des élèves, ils relativisent dans le même temps cet effet par rapport aux effets engendrés par d'autres facteurs. Leithwood et al. (2008) mettent en évidence, par exemple, que l'effet de l'activité des chefs d'établissement est moins important que celui de la nature des pratiques pédagogiques des enseignants en classe. À partir d'une analyse de différentes études quantitatives, ces auteurs estiment que l'effet du chef d'établissement ne représente que 5 à $7 \%$ dans la réussite des élèves, alors même que l'effet établissement y contribuerait pour une part variant entre 12 à $20 \%$, et l'effet classe pour plus d'un tiers. Outre le fait de relativiser les retombées des activités des chefs d'établissement sur l'amélioration de la réussite des élèves, certains travaux appellent à des méthodes d'observation ou d'analyse plus larges que celles généralement employées, la taille des échantillons étant parfois considérée comme non représentative (Spillane \& Diamond, 2007; Leithwood et al., 2008). Des travaux de synthèse pointent, par ailleurs, la faible prise en compte du contexte, notamment sociologique, des établissements scolaires et, par extension, interrogent la validité «internationale» de résultats obtenus dans le cadre de contextes nationaux par définition singuliers (Hallinger 2011a; Hallinger, Leithwood \& Heck, 2010; Tan, 2016).

\section{Difficultés à surmonter}

Le faible nombre de travaux portant sur les activités réalisées par les chefs d'établissement peut être, en partie, expliqué par les difficultés spécifiques liées à l'observation de celles-ci au quotidien. Certains auteurs (Cros, 2011; Losego, 2013) considèrent en effet que cette observation est nécessairement lacunaire et incomplète, tant ces activités sont multiples, diverses, parfois triviales (téléphoner, 
envoyer un mail, dialoguer, ...), et qu'elles ne sont pas toujours explicites du point de vue des finalités qui les sous-tendent. Dans le même ordre d'idées, Roegman, Hatch et Riehl (2012) soulignent, quant à eux, que le leadership pédagogique relève d'activités complexes tout à la fois cognitives et sociales et très largement influencées par les contingences locales. Gather Thurler et al. (2011) notent également que les activités cognitives ou relationnelles des chefs d'établissement sont nombreuses et essentielles et, pour autant, n'en restent pas moins difficilement observables. On comprend dès lors le constat selon lequel les activités des chefs d'établissement restent difficilement saisissables et descriptibles.

Cette difficulté à appréhender finement les activités des chefs d'établissement tient sans doute aussi du fait qu'elles ont la particularité d'être fragmentées et hétérogènes. Elles sont en effet marquées de façon structurelle par des interruptions très fréquentes (Barrère, 2013; Losego, 2013). À cette fragmentation temporelle, s'ajoute une multiplicité des buts visés, multiplicité pour ainsi dire inhérente à leurs missions d'encadrement mais aussi aux tensions pouvant exister entre les pratiques de gestion locale et les prescriptions qui se révèlent d'ailleurs parfois contradictoires (Ducros \& Amigues, 2014; Normand, 2015; Printy, 2008). Certains travaux scientifiques ont toutefois essayé de catégoriser les activités menées par les chefs d'établissement. Cependant, comme le soulignent Maulini et Progin (2014), les catégories produites relèvent de cadres théoriques toujours singuliers, et de ce fait, apparaissent bien souvent comme discutables. La catégorie "travail administratif» est, par exemple, fréquemment utilisée, alors même qu'aucune définition n'est réellement proposée d'un point de vue empirique dans la littérature (Losego, 2013). Pour Barrère (2008), cette difficulté à catégoriser subsiste y compris si les catégories sont construites par les chefs d'établissement eux-mêmes. Les catégories d'activité alors proposées sont en effet très largement imprégnées de subjectivité, et ce, d'autant plus fortement dans un métier où la "présentation de soi» est une compétence professionnelle, au même titre que la présentation de son établissement (Barbier, 2011). Il est à noter que dans les travaux anglophones, une catégorisation fait néanmoins référence, le "Principal Instructional Management Rating Scale» (PIMRS) proposé par Hallinger (1982/1990). Utilisée dans de très nombreux travaux universitaires (pour un recensement des travaux: Hallinger \& Wang, 2015), cette classification, est mobilisée pour la recherche universitaire et sert d'outil d'évaluation et de formation initiale et continue des chefs d'établissement dans plusieurs états (Neumerski, 2013).

\section{Perspectives de travail}

Malgré le travail de recension effectué, des questions subsistent. Il apparaît important d'y prêter attention, notamment pour tracer de nouvelles pistes en matière de travaux à entreprendre dans le domaine. Il semble ici opportun de mettre en avant la question relative à la nature de la concaténation des diverses 
activités menées par les chefs d'établissement, c'est-à-dire la question de l'articulation des différentes activités menées dans des temporalités et des registres hétérogènes. L'étude des différentes logiques intentionnelles qui sous-tendent cette imbrication d'activités nous semble particulièrement heuristique. Pour tenter d'ouvrir la réflexion sur ce point, la modélisation proposée par Livet (2005), en termes d'articulation entre les concepts d'action et d'intention, apparaît comme tout particulièrement heuristique. Cet auteur distingue en effet, pour comprendre une activité, ce qui relève de la «cible» et de la «motivation» de chacune des actions la constituant. Il utilise pour ce faire l'exemple suivant: si «se saisir d'une tasse» a pour cible la tasse, le mouvement déclenché pour s'en saisir se fera par le côté ou par le dessus, selon que la motivation est de boire dedans ou de la ranger. De la même manière pour un chef d'établissement, «valoriser une action lors d'un discours officiel» va potentiellement avoir des cibles différentes (l'enseignant porteur du projet, les parents d'élèves en opposition au projet, ...) et être portée par des motivations différentes (mettre en place de nouveaux projets, renforcer la cohésion de l'équipe pédagogique, faciliter l'inclusion des élèves en situation de handicap, ...). L'enrichissement de l'analyse rendu possible par l'usage des concepts de «cible» et de «motivation» pourrait sans doute permettre de mieux comprendre la nature indirecte de «l'effet» des activités des chefs d'établissement sur l'amélioration de la réussite des élèves. En effet, les activités engagées par ces derniers sont irriguées par des motivations diverses, entremêlées et imbriquées les unes dans les autres, mais aussi, dans le même temps, orientées vers des cibles diverses. Ce type d'analyse permettrait sans doute de mieux analyser la concaténation des diverses activités engagées. De façon complémentaire, cela permettrait de mieux comprendre les activités dites de détour, ou celles considérées, a priori, comme étrangères aux préoccupations d'apprentissage et de réussite chez les élèves.

Selon Livet (2005), il est par ailleurs possible d'étudier les actions constitutives d'une activité en y associant différents degrés d'intentionnalité. Pour l'auteur, il est ainsi possible, a minima, de distinguer l'«intention préalable» dont l'acteur a conscience avant l'activité et qui peut être considérée comme l'intention à son origine, et l'«intention en action» qui fait corps avec l'activité en cours de réalisation. De façon complémentaire, il précise que la constitution d'une intention préalable est le résultat d'une élaboration cognitive progressive, à partir de la multiplicité d'intentions en action. Cette modélisation apparaît comme particulièrement intéressante pour comprendre la concaténation des diverses activités des chefs d'établissement, par l'intermédiaire de l'étude de l'articulation entre leurs intentions préalables et leurs intentions en action. Ces concepts permettent d'éclairer les deux premiers constats de cette synthèse, et en premier lieu l'importance d'une "attention focalisée du chef d'établissement sur l'apprentissage des élèves». Cette "attention» qui fait consensus dans les travaux scientifiques pourrait sans doute être assimilée à l'intention préalable, ayant pour ainsi dire ordonné les activités des chefs d'établissement pour guider, contrôler, diriger 
l'action vers la réussite des élèves. Cette distinction entre «intention préalable» et «intention en action» apparait comme d'autant plus heuristique qu'elle permet par ailleurs de porter un regard nouveau sur le décalage pointé par plusieurs auteurs entre le nombre important de travaux dont l'objet porte, pour ainsi dire, sur "ce qu'il convient de faire et pourquoi le faire» et ceux, beaucoup moins nombreux, traitant véritablement du "comment le faire» (Hallinger, 2011b; Matringe, 2012; Mauny, 2010; Neumerski, 2013; Raelin, 2017). Les travaux les plus nombreux traitent en quelque sorte des «intentions préalables» associées aux activités des chefs d'établissement (comme par exemple: améliorer la réussite des élèves, définir une politique d'établissement, mobiliser les enseignants, ...) alors même qu'étudier les activités réellement menées, comme celles recensées dans cette revue, nécessiterait de davantage porter l'attention sur les «intentions en action».

Finalement, cette revue de littérature met en exergue la nécessité de poursuivre le travail de description et d'analyse des activités engagées par les chefs d'établissement pour améliorer la réussite des élèves. Pour ce faire, elle propose d'engager des travaux dont l'objet serait de modéliser leur concaténation par l'intermédiaire, comme introduit par exemple en amont, de l'étude des motivations qui les irriguent, des cibles qui les orientent ou encore des diverses intentions pouvant y être associées. Sur la base de cet ensemble d'observations et d'analyses, la construction d'un possible référentiel d'activités professionnelles articulées pourrait alors s'envisager et sans doute irriguer demain les actions de formation entreprises dans le cadre de la formation au leadership pédagogique des (futurs) chefs d'établissement.

\section{Bibliographie}

Baluteau, F. (2009a). Les régimes d'action des directeurs d'établissement secondaire. Carrefours de l'éducation, 28(2), 171-188.

Baluteau, F. (2009b). La division du travail pédagogique dans les établissements secondaires français. Le rôle des chefs d'établissement. Socio-logos. Revue de l'association française de sociologie, 4. Repéré à http://journals.openedition.org/socio-logos/2308.

Barbier, J. M. (2011). L’action de diriger. In J.-M. Barbier, C. Chauvigné, \& M.-L. Vitali (Éds.), Diriger : un travail (pp. 61-84). Paris, France: L'Harmattan.

Barbier, J. M., \& Durand, M. (2003). L'activité: un objet intégrateur pour les sciences sociales. Recherche et Formation, 42, 99-117.

Barrère, A. (2008). Les chefs d'établissement au travail: hétérogénéité des tâches et logiques d'action. Travail et formation en éducation, 2. Repéré à http://journals.openedition.org/ tfe/698.

Barrère, A. (2009). Les directions d'établissement scolaire à l'épreuve de l'évaluation locale. Carrefours de l'éducation, 28, 199-21.

Barrère, A. (2010). Ce que fait l'évaluation aux établissements scolaires : Une année dans un collège d'«éducation prioritaire». Ethnologie française, 40(1), 141-149.

Barrère, A. (2013). Un management bien tempéré: l'expérience des chefs d'établissement de l'enseignement secondaire français. Éducation et sociétés, 32, 21-34.

Baudrit, A., \& Rich, J. (Éds.). (2013). Modalités de leadership et indices de variations de climat dans les établissements scolaires [numéro thématique]. Recherches en éducation, 15, 7-18. 
Bossert, S., Dwyer, D., Rowan, B., \& Lee, G. (1982). The instructional management role of the principal. Educational Administration Quarterly, 18(3), 34-64.

Bravo, P.-J. (2008). Réussir le changement ? Du rôle du proviseur pour mobiliser les ressources humaines. Administration et éducation, 118, 153-166.

Brest, P. (2011). Le leadership dans les organisations publiques: le cas des chefs d'établissement de l'enseignement secondaire. Politique et management public, 28(3). Repéré à http://journals.openedition.org/pmp/4406.

Brezicha, K, Bergmark, U., \& Mitra, D. L. (2015). One size does not fit all: Differentiating leadership to support teachers in school reform. Educational Administration Quarterly, 51(1), 96-132.

Clot, Y. (2008). Travail et pouvoir d'agir. Paris, France: PUF.

Cour des Comptes. (2010). L'éducation nationale face à l'objectif de la réussite de tous les élèves. Paris, France: La Documentation française.

Cros, F. (2011). Chercheurs et dirigeants. In J.-M. Barbier, C. Chauvigné, \& M.-L. Vitali (Éds.), Diriger : un travail (pp. 35-58). Paris, France: L'Harmattan.

Day, C., Sammons, P., Leithwood, K., Hopkins, D., Harris, A., Gu, Q., \& Brown, E. (2010). Ten strong claims about successful school leadership. Nottingham, England: The National College for School Leadership.

Derouet, J. L., \& Normand, R. (2014). La question du leadership dans la tradition française: de la communauté scolaire au management. In J. L. Derouet, \& R. Normand (Éds.), La question du leadership en éducation: perspectives européennes (pp. 41-62). Louvain-la-neuve, Belgique: Thélème Academia l'Harmattan.

Ducros, C., \& Amigues, R. (2014). Le travail des chefs d'établissement: Quelques réflexions sur le travail réel. Formation et profession, 22(3), 12-23.

Dumay, X. (2009). La coordination locale du travail enseignant: une approche par le cadrage. Les Cahiers de Recherche en Éducation et Formation, 72, 1-29.

Dupriez, V. (2010). Le travail collectif des enseignants: au-delà du mythe. Travail et formation en éducation, 7, 1-10. Repéré à http://journals.openedition.org/tfe/1492.

Dutercq, Y. (2015). Les modèles contemporains de légitimité des chefs d'établissement français. Recherche et Formation, 78, 35-50.

Endrizzi L., \& Thibert, R. (2012). Quels leaderships pour la réussite de tous les élèves? Dossier d'actualité Veille et Analyses, 73, avril. Lyon, France: ENS de Lyon.

Gather Thurler, M., \& Maulini, O. (2007) L'organisation du travail scolaire: enjeu caché des réformes? Québec: Presses Universitaires du Québec.

Gather Thurler, M., Kolly Ottiger, I., Losego, P., Maulini, O., Denecker, C., Jan, A., Meyer, A., Progin, L., \& Tchouala, C. (2011). Le travail réel des directeurs d'institutions scolaires et socio-sanitaires. Une recherche conduite en Suisse romande par trois hautes écoles partenaires. HEP Lausanne, HETS Genève, Université de Genève. HEP Lausanne, HETS Genève, Université de Genève.

Gather Thurler, M., Denecker, C., \& Meyer, A. (2014). L'expérience du leadership: des tâches aux épreuves des directeurs d'établissement scolaire. In Christian Maroy (Éd.), L'école à l'épreuve de la performance. Les politiques de régulation par les résultats (pp. 33-49). Bruxelles, Belgique: De Boeck.

Gather Thurler, M., Pelletier, G., \& Dutercq, Y. (2015). Leadership éducatif? Recherche et formation, 78, 95-109.

Hallinger, P. (1982/1990). Principal instructional management rating scale. Sarasota, FL: Leading Development Associates.

Hallinger, P. (2011a). Leadership for learning: Lessons from 40 years of empirical research. Journal of Educational Administration, 50(4), 539-576.

Hallinger, P. (2011b). Progress in educational leadership. A review of three decades of doctoral studies using the principal instructional management rating scale: A lens on methodological. Educational Administration Quarterly, 47, 271-306. 
Hallinger, P. (2013). Reviewing reviews of research in educational leadership: An empirical assessment. Educational Administration Quarterly, 50, 539-576.

Hallinger, P., Leithwood, K., \& Heck, R.-H (2010). Leadership: Instructional. In P. Peterson, E. Baker, \& B. McGaw (Éds.), International Encyclopedia of Education, 5, 18-25. Oxford, England: Elsevier.

Hallinger, P., \& Wang, W. C. (2015). Assessing instructional leadership with the principal instructional management rating scale. Dordrecht, Pays-Bas: Springer.

Harris, A., \& Spillane, J. (2008). Distributed leadership through the looking glass. Management in Education, 22(1), 31-34.

Hassani, M., \& Meuret, D. (2010). La régulation de l'action des enseignants par les chefs de leur établissement. Politiques et management public, 27(4), 103-126.

IGEN-IGAENR-ESEN. (2010). Promouvoir une culture de l'évaluation et de pilotage pédagogique dans notre système éducatif. MEN. Repéré à http://www.cndp.fr/crdp-dijon/ IMG/pdf/ig_esen_eval_pilotage.pdf

Jacobson, S. (2011). Leadership effects on student achievement and sustained school success. International Journal of Educational Management, 25, 33-44.

Kools, M., \& Stoll, L. (2016). What makes a school a learning organisation? OECD Education Working Papers, 137. Paris, France: OECD Publishing.

Leithwood, K., Harris, A., \& Hopkin, D. (2008). Seven strong claims about successful school leadership. School Leadership and Management, 28(1), 27-42.

Leithwood, K., Mascall, B., Strauss, T., Sacks, R., Memon, N., \& Yashkina, A. (2007). Distributing leadership to make schools smarter: Taking the ego out of the system. Leadership and Policy in Schools, 6(1), 37-67.

Lessard, C., Kamanzi, P. C., \& Larochelle, M. (2008). La perception des politiques éducatives chez les directions d'école et les enseignants canadiens: l'influence de l'idéologie professionnelle. Sociologie et sociétés, 40(1), 93-118.

Letor, C. (2010). Moments de coopération entre enseignants: entre logiques institutionnelle, organisationnelles et professionnelles. Travail et formation en éducation, 7. Repéré à http:// journals.openedition.org/tfe/1458

Letor, C., \& Garant, M. (2014). Régulation et cadrage exercés par les directeurs de catégorie de Hautes Écoles en Fédération Wallonie-Bruxelles. In M. Garant \& C. Letor (Éds.), Encadrement et leadership. Nouvelles pratiques en éducation et formation (pp. 71-88). Bruxelles, Belgique: de Boeck.

Losego, P. (2013). Diriger: un travail en miettes? In Les «dessous» de la recherche CADRE: questions de recherche, méthodes et outils d'analyse. Actes du $85 \mathrm{e}$ séminaire de la CRoTCES (Yverdon-les-Bains, 20-21 mars 2013). Repéré à www.claceso.ch/fr/ wp-content/uploads/2015/03/Mise_Crotces.pdf.

Livet, P. (2005). Qu'est-ce qu'une action? Paris, France: Vrin.

MacBeath, J., \& Cheng, Y.C. (2008). Leadership for learning: International perspectives. Rotterdam, Pays-Bas: Sense Publishers.

Matringe, G. (2012). Les évolutions du métier de chef d'établissement. Constat, enjeux \& perspectives. Revue internationale d'éducation de Sèvres, 60, 35-44.

Maulini, O., \& Progin, L. (2013). La conception du rôle et le travail espéré. Les «dessous» de la recherche CADRE: questions de recherche, méthodes et outils. Actes du 85e séminaire de la CRoTCES (Yverdon-les-Bains, 20-21 mars 2013). Repéré à www.claceso.ch/fr/ wp-content/uploads/2015/03/Mise_Crotces.pdf.

Maulini, O., \& Progin, L. (2014). De l'idéal du rôle aux dilemmes du travail réel. In J. L. Derouet \& R. Normand (Éds.), La question du leadership en éducation. Perspectives européennes (pp. 219-234). Louvain-la-Neuve, Belgique: Acadamia-L'Harmattan.

Mauny, C. (2010). Formation professionnelle et mutation identitaire: le cas des chefs d'établissement français. Éducation et francophonie, 38(1), 72-91. 
Muijs, D. (2011). Leadership and organisational performance: From research to prescription? International Journal of Educational Management, 25, 45-60.

Muller, F., \& Normand, R. (2013). La grande transformation de l'école? Les clés du changement. Paris, France: ESF.

Murphy, J., Vriesenga, M., \& Storey, V. (2007). 1979-2003: An analysis of types of work, methods of investigation, and influences. Educational Administration Quarterly, 43, 612-628.

Neumerski, C. (2013). Rethinking instructional leadership, a review what do we know about principal, teacher, and coach instructional leadership, and where should we go from here? Educational Administration Quarterly, 49(2), 310-347.

Normand, R. (2015). French principals in Secondary Education: Towards new roles and responsibilities? ECPS Journal, 11, 135-152.

Normand, R., \& Derouet, J.-L. (2011). Évaluation, développement professionnel et organisation scolaire. Revue Française de Pédagogie, 174, 5-20.

Obin, J. P. (2012). La fin des notes pour les enseignants? Cahiers pédagogiques, 494. Repéré à http://www.cahiers-pedagogiques.com/La-fin-des-notes-pour-les-enseignants.

OCDE. (2014). Résultats de TALIS 2013: une perspective internationale sur l'enseignement et l'apprentissage. Paris, France: Éditions OCDE.

Pelletier, D., Collerette P., \& Turcotte G. (2015). Les pratiques de gestion des directions d'école secondaire sont-elles liées à la réussite des élèves? Revue canadienne de l'éducation, $38(1), 1-23$

Perrenoud, O. (2015). Les chefs d'établissement peuvent-ils faire évoluer l'organisation du travail et les pratiques pédagogiques? Formation et Profession, 23(3), 60-69.

Poirel, E., \& Yvon, F. (2012). Le leadership distribué: l'épreuve des réformes éducatives. Éducation et Francophonie, 40(1), 94-118.

Pont, B., Nusche, D. P., \& Moorman, H. (2008). Améliorer la direction des établissements scolaires: volume 1: politiques et pratiques. Paris, France: OCDE.

Printy, S. (2008). Leadership for teacher learning: A community of practice perspective. Educational Administration Quarterly, 44(2), 187-226.

Printy, S., Marks, H. M., \& Bowers, A. J. (2009). Integrated leadership: How principals and teachers share instructional influence. Journal of School Leadership, 19(5), 504-532.

Progin, L. (2015). Le leadership: un concept et objet de formation tabou pour les chefs d'établissement débutants? Recherche et Formation, 78, 69-80.

Progin, L., \& Gather Thurler, M. (2010). Le leadership pédagogique: un levier pour transformer l'organisation du travail au sein des établissements scolaires? Actes du congrès de l'Actualité de la recherche en éducation et en formation (AREF), Université de Genève, 13 au 16 septembre.

Progin, L., \& Gather Thurler, M. (2012). Le leadership pédagogique: un levier pour transformer l'organisation du travail au sein des établissements scolaires? Recherches en Éducation, 4, 42-54.

Progin, L., \& Gather Thurler, M. (2017). Devenir chef d'établissement: le désir de leadership à l'épreuve de la réalité. Berne, Suisse: Peter Lang.

Raelin, J. (2017). Leadership-as-practice: Theory and application-An editor's reflection. Leadership, 13(2), 215-221.

Robinson, V. M. J., Lloyd, C. A., \& Rowe, K. J. (2008). The impact of leadership on student outcomes: An analysis of the differential effects of leadership types. Educational Administration Quarterly, 44(5), 635-674.

Robinson, V. (2010). From instructional leadership to leadership capabilities: Empirical findings and methodological challenges. Leadership and Policy in Schools, 9(1), 1-26.

Roegman, R., Hatch, T., \& Riehl, C. (2012). Le leadership en des temps incertains. Revue internationale d'éducation de Sèvres, 60, 44-54. 
Salamé Sala, J., \& Dutercq, Y. (2015). Convergence et spécificités nationales de la direction des établissements scolaires en Europe. Recherche et Formation, 78(1), 111-117.

Sergi V. (2016). Who's leading the way? Investigating the contributions of materiality to leadership-as-practice. In: J.-A. Raelin (Éd.), Leadership-as-practice: theory and application (pp.110-131). New York, NY: Routledge.

Spillane, J., \& Diamond, J. B. (2007). Distributed leadership in practice. New York, NY: Teachers College Press.

Strauss, A., \& Corbin, J. (1990). Basics of qualitative research: Grounded theory procedures and techniques. Newbury Park, CA: Sage Publications.

Supovitz, J., Sirinides, P., \& May, H. (2010). How principals and peers influence teaching and Learning. Educational Administration Quarterly, 46(1), 31-56.

Tan, C. Y. (2016). Examining school leadership effects on student achievement: the role of contextual challenges and constraints. Cambridge Journal of Education, 48(1), 21-45.

Tourmen, C., Mayen, P., \& Samrany, L. (2011). Qualifier, une activité méconnue des dirigeants. In J.-M. Barbier, C. Chauvigné, \& M.-L. Vitali (Éds.), Diriger : un travail (pp. 119-148). Paris, France: L'Harmattan.

Mots-clés: Chef d'établissement, leadership pédagogique, leadership, réussite des élèves, activité

\section{Welche Aktivitäten werden von Schulleitern im Rahmen der Bildungsführung tatsächlich durchgeführt? Literaturreview}

\section{Zusammenfassung}

Dieser Artikel gibt einen überblick über die Literatur zu täglichen Aktivitäten von Schulleitern/innen bei der Ausübung von Führungsmassnahmen. Es ist wichtig zu betonen, dass der «Schulleitungseffekt» zur Verbesserung des Schülererfolgs zwar gut untersucht zu sein scheint, aber hinsichtlich der tatsächlich eingesetzten täglichen Aktivitäten in Schulen wenig diskutiert wird. Auf der Grundlage der Beschreibung der untersuchten Hauptaktivitäten, z. B. Aktivitäten in Bezug auf Schulauftrag, Mobilisierung und Lehrerbildung, Einfluss auf pädagogisches Handeln oder der strategischen Gestaltung der Schulstruktur und -organisation, eröffnet der Artikel neue Sicht- und Arbeitsweisen.

Schlagworte: Schulleiter, Bildungsführung, Führung, Schülererfolg, Aktivität 


\section{Quali sono le attività effettive dei dirigenti scolastici nel contesto della leadership educativa? Rassegna della letteratura}

Riassunto

Questo articolo riporta una rassegna della letteratura sulle attività quotidiane dei dirigenti scolastici nell'esercizio della loro leadership pedagogica. La rassegna permette di evidenziare come l'effetto della "leadership della scuola» sul miglioramento del successo degli studenti risulti ben studiato, mentre rimane poco discussa tale leadership in relazione alle attività quotidiane effettivamente implementate nelle scuole. Sulla base della descrizione delle principali attività studiate, come quelle relative alla missione scolastica, alla mobilitazione e alla formazione degli insegnanti, all'influenza sulle pratiche pedagogiche o all'uso strategico della struttura e dell'organizzazione scolastica, l'articolo apre nuove strade di lavoro.

Parole chiave: Preside della scuola, leadership educativa, leadership, successo degli studenti, attività

\section{What activities are actually engaged by school leaders in leadership for learning? Literature review}

\section{Abstract}

This article reports on the literature on the day-to-day activities of school leaders in exercising leadership for learning. It helps to highlight that while the «school leadership effect» on the improvement of student success appears to be well studied, it remains little discussed in terms of actually deployed daily activities in schools. On the basis of the description of the main activities studied, such as those relating to school mission, mobilization and training of teachers, influence on pedagogical practices, or strategic use of school's structure and organization, the article opens new avenues of work.

Key words: School principal, educational leadership, leadership, student success, activity 
Sylvie Bos, Chef d'établissement et Doctorante en Sciences de l'Education et de la Formation. Ses préoccupations de recherche portent sur l'activité des chefs d'établissement en contexte ordinaire de travail et sur les modalités de leur formation professionnelle.

UMR EFTS, Université de Toulouse Jean Jaurès, Maison de la recherche 5, allées Antonio-Machado, F-31 058 Toulouse Cedex 9

E-Mail: sylvie.bos@univ-tlse2.fr

Sébastien Chaliès, Professeur des Universités à l'École Supérieure de l'Éducation et de la Formation de Toulouse Midi-Pyrénées (Université de Toulouse - France). L'ensemble de son travail scientifique a pour finalité la construction et le développement d'un "programme de recherche» singulier mené en sciences de la formation d'adultes. Mené en anthropologie culturaliste, ce programme porte sur la construction du sujet professionnel en formation. D'un point de vue épistémologique, ce programme emprunte à la philosophie analytique, à la sociologie de la connaissance et à l'anthropologie culturelle. Les principales problématiques travaillées au cœur de ce programme sont relatives au principe de l'alternance entre travail et formation au travail, à la place de l'ostension/ prescription et de la réflexivité en formation, à la construction de la santé au et par le travail ou encore à la créativité professionnelle.

ESPE de Toulouse - Midi-Pyrénées Université de Toulouse, Site de Rangueil, 118 Route de Narbonne, F-31078 Toulouse

E-Mail: Sebastien.chalies@univ-tlse2.fr 